\title{
Avance de tesis doctoral: Aceros de alta resistencia como refuerzo en estructuras de hormigón
}

\section{Doctoral Thesis progress: High Strength Steels as reinforcement in concrete structures}

Presentación: 06/10/2020

\section{Doctorando:}

\section{María José Castillo}

Tecnología de Procesos, Centro de Desarrollo y Tecnología de Materiales, Facultad Regional San Nicolás San Nicolás, Universidad Tecnológica Nacional -Argentina

mariajose.castillo@nexo.unnoba.edu.ar

\section{Director/a:}

\section{Hernán Svoboda}

\section{Co-director/a:}

\section{María del Carmen Andrade}

\section{Resumen}

Este trabajo resume el trayecto de trabajo experimental vinculado a la temática de la tesis en curso. El objetivo es analizar el efecto de los parámetros de soldadura por resistencia en barras de acero de uso comercial y aceros DP obtenidos mediante tratamiento térmico de austenización parcial. En primera instancia, se resumió la puesta a punto de equipos y técnicas de caracterización. Posteriormente, se evaluó el comportamiento de uniones soldadas por proyección en barras ATR 500 N y en aceros DP obtenidos por tramitamiento térmico de austenización parcial.

Palabras clave: aceros de alta resistencia, soldadura por proyección, propiedades mecánicas, hormigón armado

\begin{abstract}
This paper talk about the experimental work developed in the thesis in progress. The aim of this paper is analyzing the effect of resistance welding parameter's on commercial and, Dual Phase bars obtained through partial austenitizing heat treatment. In first place, it was summarized the commissioning of equipment and characterization techniques. After that, it was evaluated the behavior of Projection welded joints on ATR $500 \mathrm{~N}$ and Dual Phase bars obtained through partial austenitizing heat treatment.
\end{abstract}

Keywords: high strength steel, projection welding, mechanical properties, reinforced concrete

\section{Introducción}

El hormigón es un material compuesto que, una vez endurecido, se asemeja a una piedra artificial. En general, todos los hormigones y, especialmente los convencionales, están formados por áridos, cemento y agua. Además de los componentes 
principales, hay otros componentes secundarios como, por ejemplo, aditivos, fibras, cargas, armaduras metálicas. En el hormigón armado, la armadura constituye uno de los componentes fundamentales (Menéndez Méndez, Esperanza (2014)). Diversos tipos de aceros pueden utilizarse como refuerzo en estructuras de hormigón. En nuestro país, las barras ADN 420 y ADN 420S (IRAMIAS U 500207 (2004), IRAM-IAS U 500528 (2004)), son frecuentemente utilizadas por sus características mecánicas y buena soldabilidad. Para la fabricación de mallas o estructuras tipo TRILOGIC, se utilizan productos especificados por la norma IRAMIAS U500-26 (IRAM-IAS U 50026 (2016)). Las características plásticas del acero tienen gran importancia ya que evitan las roturas frágiles, redistribuyen esfuerzos y aumentan la capacidad de disipar energía bajo cargas dinámicas, como es el caso de las cargas sísmicas. En los últimos años, se ha desarrollado una familia de aceros denominada Aceros Avanzados de Alta Resistencia (AHSS: Advanced High Strenght Steels), que presentan propiedades optimizadas respecto de los materiales convencionales. Dentro de esta familia se encuentran aceros como los Dual Phase (DP), cuya microestructura está constituida principalmente por partículas de martensita dispersas en una matriz de ferrita, donde dicha matriz garantiza una alta conformabilidad y la martensita proporciona alta resistencia. Los aceros DP se caracterizan por su comportamiento de fluencia continua, baja tensión de fluencia, relación favorable entre tensión de fluencia y tensión máxima (aproximadamente 0,5) y un alto nivel de elongación con un coeficiente de endurecimiento por trabajado elevado (Kelesternur et al, (2009)). Las propiedades previamente mencionadas de los aceros DP, sugieren que podrían presentar un fuerte impacto en cuanto a su uso en estructuras sismo-resistentes de hormigón armado, debido a su alta tasa de endurecimiento por deformación y su alta capacidad de absorción de energía. En la actualidad, los estudios sobre el efecto del tratamiento térmico de templado intercrítico sobre las propiedades mecánicas de los aceros DP son limitados. Asimismo, hasta la fecha, las aplicaciones más destacadas de estos materiales como elementos estructurales se encuentran asociadas a chapa, principalmente en la industria automotriz. Existiendo muy poca información respecto al empleo de estos aceros en el campo de la construcción (Maffei et al, (2007)). En relación a la conformación de mallas de acero, éstas son en general electrosoldadas a través de procesos de soldadura por resistencia (RW: Resistance Welding), donde el calor generado para producir la unión surge de la resistencia que oponen las piezas de trabajo al paso de la corriente eléctrica, bajo la acción de presión mecánica. Específicamente, para la unión de barras electrosoldadas, donde el número de soldaduras a realizar al mismo tiempo es elevado, se utiliza el proceso de soldadura por proyección, (PW; Projection Welding) el cual es una variante de RW. En este caso, el flujo de corriente es concentrado en puntos de contacto entre las partes a ensamblar. Estas extensiones son utilizadas para concentrar el calor en el punto de contacto. La operación de soldadura PW implica la aplicación coordinada de corriente eléctrica y presión mecánica donde, la secuencia de operación debe, en primer lugar, generar suficiente calor para llevar un volumen confinado del metal a la temperatura de forjado. Luego, el metal se enfría bajo presión (Zhang et al, H (2006)). El objetivo de este trabajo es analizar el efecto de los parámetros de soldadura por resistencia en barras de acero de uso comercial y aceros DP obtenidos mediante tratamiento térmico de austenización parcial.

\section{Desarrollo}

\section{Primera Etapa: puesta a punto de técnicas de caracterización y equipos}

En la primera etapa se trabajó en la puesta a punto de las diferentes técnicas de trabajo. Particularmente, en el equipo de soldadura, evaluación de las variables involucradas en los procesos de soldadura y caracterización de las uniones soldadas. Para la ejecución de esta actividad se utilizaron barras ATR 500N comerciales (IRAM-IAS U 50026 (2016)), de 4 mm de diámetro y $100 \mathrm{~mm}$ de longitud, dispuestas en forma transversal. Sobre éstas se efectuaron uniones soldadas mediante el proceso PW, variándose los ciclos de soldadura (4, 6, 8 y 10 ciclos), la fuerza de soldadura (desde 750 a $2500 \mathrm{~N}$ ) y la corriente promedio $(4,5$; 5,5 y 6,5 kA). En relación a la macroestructura, se observó, en general, para las configuraciones de bajo tiempo de soldadura y baja corriente con fuerza intermedia, un bajo nivel de indentación del alambre superior respecto del inferior, sin observarse flujo plástico significativo entre ambos alambres y una zona de unión pequeña (Figura 1a). Asimismo, aquellas uniones realizadas con un alto tiempo de soldadura (10 ciclos) y corriente y fuerza intermedias, el nivel de indentación resultó excesivo, con una zona afectada por el calor (ZAC) significativamente más grande que en los casos anteriores. Por otro lado, dicho incremento en el tiempo, ocasionó un sobrecalentamiento, provocando un flujo excesivo de material fuera de la unión (Dent et al, (1996)) (Figura $1 b)$. 

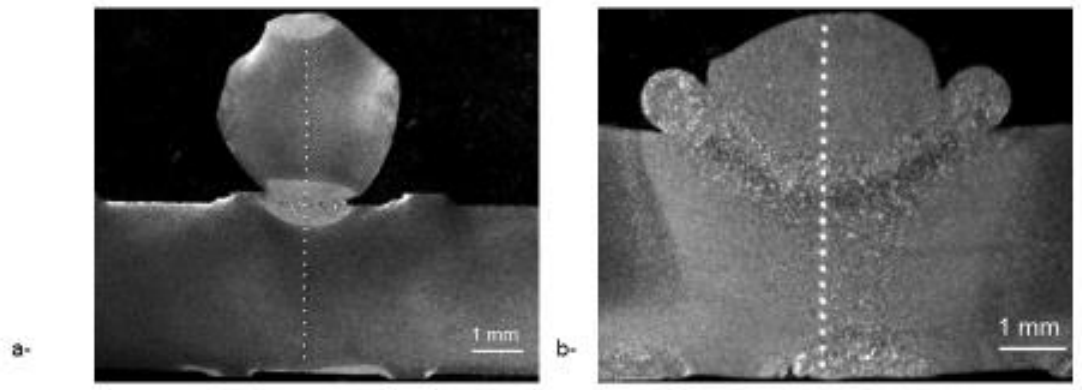

Figura 1: Macrografías; (a) 4 ciclos, 1350 N, 4,5 kA; (b) 10 ciclos, 1000 N, 5,5 kA

$\mathrm{Al}$ evaluar los valores mínimo, máximo y promedio en los barridos de microdureza realizados sobre probetas soldadas con presión y corriente intermedias para los distintos tiempos de soldadura, pudo observarse un incremento de dichos valores al descender el tiempo de soldadura, consecuencia del menor calor aportado y, de la formación de una fracción más alta de fases duras asociadas a la mayor velocidad de enfriamiento (Figura 2a). En el caso de aquellas probetas soldadas con valores crecientes de corriente promedio, se observó una disminución de los valores de microdureza al incrementarse la corriente promedio utilizada. Esto estaría asociado a la menor velocidad de enfriamiento vinculada al mayor aporte térmico (Bohr et al, J (2007)) lo que produce la formación de mayor fracción de fases ferríticas blandas (Figura 2b). En los ensayos de resistencia al corte, se observaron mayores cargas de rotura para las probetas soldadas con mayor corriente promedio (5,5 kA) y mayor tiempo de soldadura, debido a un mayor aporte de calor que mejora la resistencia mecánica de la junta (Akkas et al, (2013)).
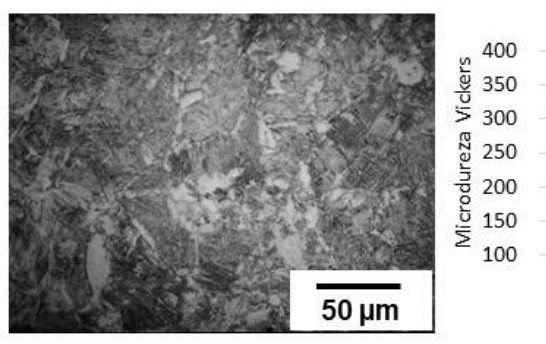

$\multimap$ MÁXIMO

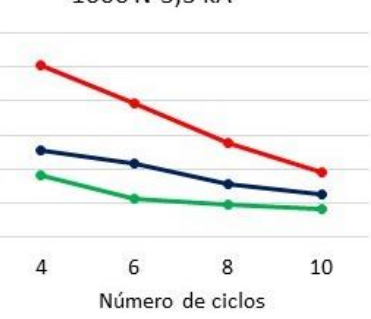

$\rightarrow$ PROMEDIO $\rightarrow$ MÍNIMO

(a)

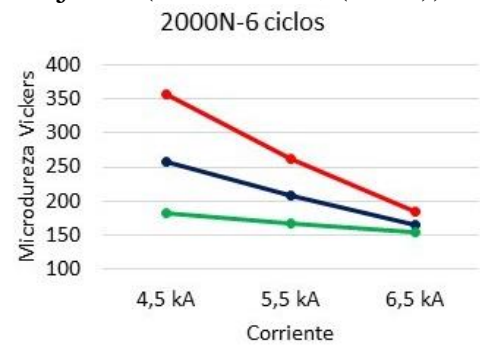

$\rightarrow$ MÁXIMO $\rightarrow$ PROMEDIO $\longrightarrow$ MÍNIMO

(b)

Figura 2: (a) Resultados de microdureza (1000 N-5,5 kA) y micrografía de la interfase correspondiente a fase duras; (b) Resultados de microdureza (2000 N-6 ciclos) y micrografía de la interfase correspondiente a fase blandas

\section{Segunda etapa: soldadura por resistencia de barras ATR de 8 mm de diámetro}

En esta etapa se trabajó en la soldadura por resistencia de barras ATR $500 \mathrm{~N}$ comerciales, de $8 \mathrm{~mm}$ de diámetro. Las uniones fueron efectuadas mediante el proceso de soldadura PW. Al igual que en la etapa 1, se evaluó la influencia de la corriente de soldadura, el tiempo de soldadura y la fuerza aplicada. Los parámetros utilizados, fueron definidos en función de los resultados obtenidos previamente: tiempo de soldadura: 10, 20, 30 y 40 ciclos, fuerza de soldadura: 1350 y $2000 \mathrm{~N}$ y corriente: 4,5; 5,5 y 6,5 kA. A escala macrográfica, pudo verse que se tiene una mayor indentación de una de las barras en la otra, al aumentar la corriente y la fuerza de soldadura (Figura 3). Asimismo, la dureza máxima disminuye debido a la menor velocidad de enfriamiento. Se verificó el cumplimiento de los requisitos establecidos por la norma IRAM-IAS U 50006 (2016). La carga de rotura aumentó con la fuerza, la corriente y el tiempo de soldadura. A partir de estos resultados se logró optimizar el procedimiento de soldadura, alcanzado una alta carga de rotura $(10.000 \mathrm{~N})$ con bajo tiempo de soldadura y baja fuerza, empleando una elevada potencia eléctrica lo que permite incremental la vida de los electrodos y la productividad. 


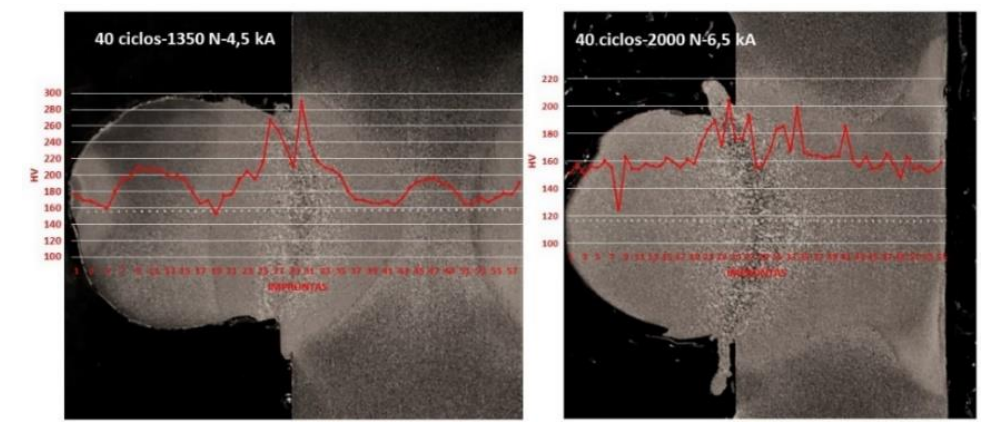

Figura 3: Macrografías y perfil de microdureza para dos de las probetas soldadas

\section{Tercera etapa: desarrollo de aceros DP}

En esta etapa se trabajó en el desarrollo de aceros DP. Se partió de un acero comercial del tipo ADN $420 \mathrm{~S}$ de $8 \mathrm{~mm}$ de diámetro (IRAM-IAS U 500207 (2004)). Dicho material fue sometido a un tratamiento térmico de normalizado a $850{ }^{\circ} \mathrm{C}$, durante 20 min y enfriamiento al aire. Posteriormente, se realizó un tratamiento térmico de austenización parcial a $730{ }^{\circ} \mathrm{C}$ durante 30 min, seguido de un temple en agua, de forma de obtener la estructura dual ferrita-martensita. Posteriormente, barras de $150 \mathrm{~mm}$ de longitud del acero DP obtenido, fueron soldadas mediante el proceso de soldadura PW, disponiéndolas en forma ortogonal, con la soldadura en el centro de la longitud. Se utilizaron electrodos planos de cobre (d=15,8 mm) refrigerados con un caudal de agua de 6 l/min en un equipo de soldadura por resistencia ROLASOL de $30 \mathrm{kVA}$. Se utilizaron dos niveles de corriente: 4, 5 kA y 6,5 kA y tres tiempos de soldadura: 20, 30 y 40 ciclos. La presión ejercida por los electrodos fue de $2000 \mathrm{~N}$. El tiempo de presoldadura fue de 50 ciclos y el de post-soldadura de 10 ciclos. Sobre cortes transversales de cada una de las uniones soldadas, se realizó una caracterización macro y microestructural empleando microscopía óptica. Se realizaron barridos de microdureza Vickers con 300 gf (HV0,3) de carga sobre la sección mayor de una de las varillas soldadas. Las improntas fueron realizadas cada 0,25 mm (ASTM E384 (1999)). Por último, se efectuaron ensayos de resistencia al corte (IRAM-IAS U 50006 (2016)). Macroestructuralmente se observó que al aumentar el tiempo y la corriente de soldadura, la zona de unión aumentó significativamente, así como el tamaño de la ZAC. El nivel de indentación o asentamiento, el cual es calculado a partir de la altura de la junta antes y luego de ser soldadas (Dent et al, (1996)) es otro aspecto de relevancia, observándose que también aumentó con el aporte de calor (tiempo y corriente de soldadura). Los valores de dureza medidos en la zona asociada a la soldadura disminuyeron al aumentar el tiempo y la corriente de soldadura, ya que el mayor aporte de calor, genera una disminución de la velocidad de enfriamiento (Bohr et al, (2007)). Por otro lado, al utilizar parámetros de soldadura mayores, el nivel de indentación aumentó, con una ZAC de mayor tamaño generada por el sobrecalentamiento, lo que provocó, además, desplazamiento de material en exceso hacia los laterales. En general, cuando el material presenta mayor dureza, entonces menor es el nivel de asentamiento, encontrándose una buena correlación entre ambos valores (Figura 4). En relación a los ensayos de resistencia al corte, el valor de resistencia mínimo establecido por norma (IRAM-IAS U 50006 (2016)) es de 7500 N. En este sentido, pudo observarse que las probetas soldadas con mayor nivel de corriente, cumplieron en todos los casos con los requisitos establecidos por norma. Las probetas soldadas con bajo nivel de corriente y bajo número de ciclos, no alcanzaron el mínimo establecido, esto estaría asociado al bajo nivel de indentación de una barra en la otra, por la falta de calor y la mayor dureza de los aceros DP, produciendo una longitud de unión pequeña.
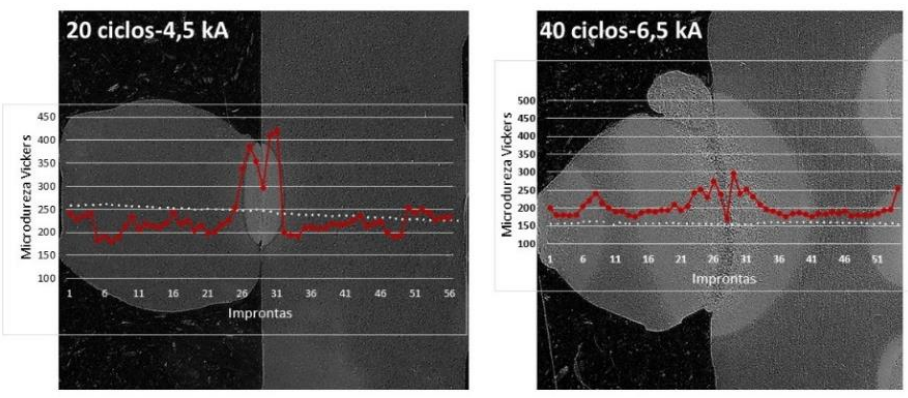

Figura 4: Macrografías y perfiles de microdureza en dos condiciones de soldadura. 


\section{Cuarta etapa: comparación de soldaduras por proyección barras ATR-barras DP}

En esta etapa de trabajo se compararon los resultados obtenidos en las etapas 2 y 3 (Figura 5). Como material de referencia se utilizó un acero comercial ATR $500 \mathrm{~N}$ de $8 \mathrm{~mm}$ de diámetro (analizado en la etapa 2). Ambos materiales cumplen con los requisitos para un producto ATR 500. El acero DP730 posee una resistencia a rotura, dureza y alargamiento superiores a los medidos para el ATR comercial, mientras que la tensión de fluencia es menor. Asimismo, puede observarse que en el DP la relación entre tensiones es significativamente mayor, asociado a la capacidad de endurecimiento por deformación (Tabla 1).

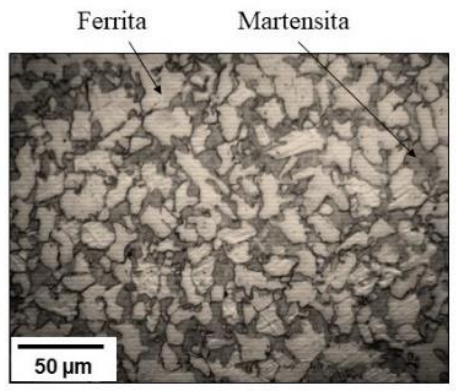

(a)

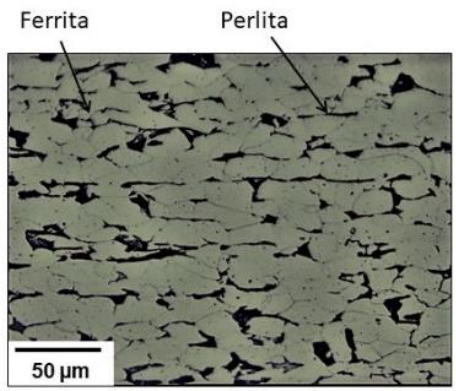

(b)

Figura 5: Microestructura de aceros DP y ATR

\begin{tabular}{|l|c|c|c|c|c|c|}
\hline Probeta & HV $_{\mathbf{1 k g}}$ & $\mathbf{R p}(\mathbf{M P a})$ & $\mathbf{R r}(\mathbf{M P a})$ & $\mathbf{A ~ ( \% )}$ & $\mathbf{R R / R p}$ & Microest $^{(*)}$ \\
\hline DP & 250 & 563 & 833 & 8 & 1,48 & $70 \mathrm{~F}-30 \mathrm{M}$ \\
\hline ATR & 240 & 670 & 708 & 7 & 1,06 & F def. frío \\
\hline Req. min. ${ }^{(*)}$ & - & 500 & 550 & 6 & - & - \\
\hline
\end{tabular}

${ }^{(*)}$ F: Ferrita; M: Martensita; ${ }^{(* *}$ Según Norma (IRAM-IAS U 500026 (2016))

Tabla 1: Propiedades mecánicas de las barras de acero DP y ATR, así como los requerimientos establecidos por norma para este producto

Al observar las Figura 3 y 4, previamente analizadas, puede notarse que la probeta ATR soldada con parámetros bajos, muestra un bajo nivel de asentamiento, esta situación es comparable con lo observado en la probeta DP, cuyos parámetros de soldadura son similares. Adicionalmente, no se observa flujo plástico significativo y la zona de unión resulta pequeña. Por otro lado, para los aceros DP, en general, se tienen durezas superiores a las obtenidas en los aceros ATR. Esta observación se asocia al mayor contenido de carbono del acero DP, respecto a los ATR, por lo que, en la zona de alta temperatura, donde se alcanza la austenización del material, existe una mayor tendencia a formar estructuras martensíticas, de mayor dureza (Tabla 2).

\begin{tabular}{|l|l|l|l|l|l|l|l|l|l|l|l|l|}
\hline PROB. & DP24 & DP34 & DP44 & DP26 & DP36 & DP46 & ATR24 & ATR34 & ATR44 & ATR26 & ATR36 & ATR46 \\
\hline (")ASENT. (\%) & 1,1 & 3,7 & 6,7 & 7,8 & 10,4 & 13,7 & 2,4 & 5,3 & 7,1 & 9,3 & 12,4 & 19,7 \\
\hline HV $_{0,3}$ ZAC & 352 & 315 & 259 & 267 & 240 & 211 & 301 & 223 & 200 & 274 & 193 & 177 \\
\hline
\end{tabular}

Por otro lado, pudo determinarse que, para bajos niveles de corriente, los aceros ATR presentaron mayores valores de resistencia respecto a los aceros DP. Sin embargo, cuando la corriente aumenta, este comportamiento se invierte, encontrándose valores de resistencia muy superiores para los aceros DP (Figura 6a).

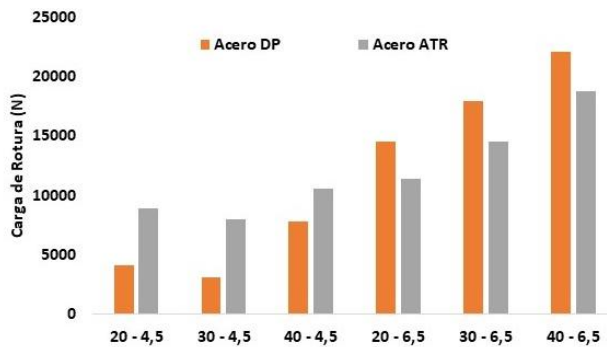

(a) Probetas

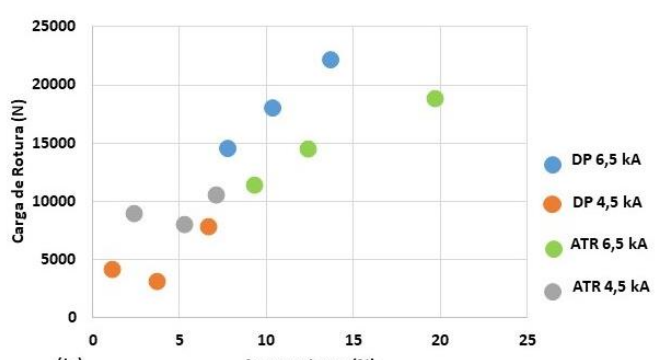

(b)

Figura 6: (a) Comparación entre la resistencia de los ATR y DP. (b) Carga de rotura en función del asentamiento para las distintas probetas soldadas 
En relación al nivel de asentamiento, se observó que éste aumenta, al incrementarse la carga de rotura, dado que se tiene una mayor longitud de unión. Asimismo, para la menor corriente $(4,5 \mathrm{kA})$ se ve que las probetas soldadas con ATR tienen mayor resistencia que las soldadas con DP, esto está asociado a que las ATR presentan mayor asentamiento, debido a la menor dureza. Para la mayor corriente $(6,5 \mathrm{kA})$ se tienen mayores valores de resistencia a rotura, siendo superiores las obtenidas para los aceros DP, si bien los valores de asentamiento son menores que para la probeta correspondiente en ATR. En este caso, dado que el nivel de asentamiento es elevado, lo que estaría controlando la resistencia de la unión es la dureza en la zona de la soldadura, siendo mayor para el caso de los DP.

\section{Conclusiones}

Este trabajo resume el trayecto de trabajo experimental vinculado a la temática de la tesis en curso. En primera instancia, se trabajó en la puesta a punto de técnicas y equipos, encontrándose relaciones vinculantes entre los parámetros de soldadura por proyección y las propiedades de las uniones soldadas resultantes. Estos resultados fueron utilizados en etapas posteriores de trabajo, donde se analizó el comportamiento de barras tradicionalmente utilizadas en el conformado de mallas electrosoldadas, frente al de aceros DP obtenidos por tratamiento térmico de aceros ADN $420 \mathrm{~S}$. En este caso, los resultados hallados, demostraron que para alta corriente, los aceros DP alcanzaron valores de hasta $22000 \mathrm{~N}$, mayores a los obtenidos para los ATR. Además, un aumento en la corriente y tiempo de soldadura incrementó la indentación de una barra en otra, aumentando, de este modo, la longitud de la unión. Por último, en cuanto al proceso de fabricación, si bien en la ruta alternativa planteada en el presente trabajo se introduce la necesidad de realizar tratamiento térmico continuo, se elimina el proceso de laminación en frío asociado a la fabricación de los ATR.

\section{Referencias}

Akkas, N., Varol, F. \& Ferik, E. (2013). Effect of Welding Current on Mechanical Properties of Welding Joints in S235JR (Cu) steel Sheets in Rersistance Spot Welding. Proceedings of 3rd International Congress APMAS, 24-28.

ASTM E384 - 99. Standard Test Method for Microindentation Hardness of Materials. 100 Barr Harbor Drive, PO Box C700, West Conshohocken, PA 19428-2959, United States. 1999.

Bohr, J., Brafford, W., Daumeyer, G., Kelly, B., Kelly, D., Kimchi, M. \& Zhang, H. (2007). Welding Projection. En R. O’ Brien (Ed), Welding Handbook (9 ed., Vol III, pp. 49-71). American Welding Society.

Dent, P., Bohr, J., Gasser, R., Gerken, J., Hallum, D., Lee, J., McCauley, R., Oris, D., Oyler G., Shieh, W. \& Wu, K. (1996). Soldadura de puntos, de costura y de proyección en R. O' Brien (Ed), Manual de Soldadura (8 ed., Vol. II, pp.531-580). American Welding Society.

IRAM-IAS U 500 06. Mallas de alambres de acero soldados para armadura en estructuras de hormigón. Perú 552/6, C1068AAB, Buenos Aires, Argentina. 2016.

IRAM-IAS U 500 26. Alambres de acero para armadura en estructuras de hormigón. Perú 552/6, C1068AAB, Buenos Aires, Argentina. 2016.

IRAM-IAS U 500 207. Barras de acero conformadas de dureza natural soldables, para armadura en estructuras de hormigón. Perú 552/6, C1068AAB, Buenos Aires, Argentina. 2004.

IRAM-IAS U 500 528: barras de acero conformadas de dureza natural, para armadura en estructuras de hormigón. Perú 552/6, C1068AAB, Buenos Aires, Argentina. 2004.

Kelesternur, O., Halidun Kelestemur M. \& Yildiz, S. (2009). Improvement of Mechanical Properties of Reinforcing Steel Used in the Reinforced Concrete Structures. Journal of Iron and Steel Research International, 16 (3), 55-63.

Maffei, B., Salvatore, W. \& Valentini, R. (2007). Dual-phase steel rebars for high-ductile r.c. structures, Part 1: Microstructural and mechanical characterization of steel rebars. Engineering Structures, 29, 3325-3332.

Menéndez Méndez, Esperanza. (2014). Interacción del hormigón con el medioambiente. Acciones físico-químicas [Cursos avanzados Eduardo Torroja "Durabilidad, Rehabilitación y Sostenibilidad].

Zhang, H., Hang, H. \& Senkara, J. (2006). Resistance Welding: Fundamentals and Applications. CRC Press. 